\title{
ASYMPTOTIC BEHAVIOR OF SOLUTIONS OF NONLINEAR FUNCTIONAL DIFFERENTIAL EQUATIONS
}

\author{
JONG SOO JUNG \\ Department of Mathematics, Dong-A University \\ Pusan 607-714, Korea \\ JONG YEOUL PARK \\ Department of Mathematics, Pusan National University \\ Pusan 609-735, Korea \\ and \\ HONG JAE KANG \\ Department of Mathematics, Graduate School \\ Dong-A University \\ Pusan 607-714, Korea
}

(Received November 24, 1992)

\begin{abstract}
Using the properties of almost nonexpansive curves introduced by B. Djafari Rouhani, we study the asymptotic behavior of solutions of nonlinear functional differential equation $d u(t) / d t+A u(t)+G(u)(t) \ni f(t)$, where $A$ is a maximal monotone operator in a Hilbert space $H, f \in L^{1}(0, \infty: H)$ and $G: C\left([0, \infty): \overline{D(A))} \rightarrow L^{1}(0, \infty: H)\right.$ is a given mapping.
\end{abstract}

KEY WORDS AND PHRASES. Almost nonexpansive curve, maximal monotone operator, generalized solution, asymptotic behavior.

1991 AMS SUBJECT CLASSIFICATION CODES. 47H20, 34G20, 47H05, 47H09.

\section{INTRODUCTION.}

Let $H$ be a real Hilbert space. We consider the initial value problem

$$
\begin{aligned}
& \frac{d u(t)}{d t}+A u(t)+G(u)(t) \ni f(t), \quad 0<t<\infty \\
& u(0)=x,
\end{aligned}
$$

where $A$ is a maximal monotone (possibly multivalued) operator defined on a subset $D(A)$ contained in $H, x \in \overline{D(A)}, f \in L_{l o c}^{1}([0, \infty): H)$ and $G$ is a given mapping

$$
G: C([0, T]: \overline{D(A)}) \rightarrow L^{1}(0, T: H), \text { for all } T>0 .
$$

Problems of the type (1.1) have been considered by many authors (see [1]-[7]). Crandall and Nohel [4] treated the problem in connection with the study of a related nonlinear Volterra equation, and obtained the existence result of generalized solution of (1.1), provided that $G$ satisfies a Lipschitz type condition. In particular, under some suitable hypotheses on $A$ and $G$, Aizicovici [1] obtained nice asymptotic results of generalized solutions of (1.1), which are the 
natural analogs of the evolution case (i.e., $G \equiv 0$ ). Using the convergence condition of Pazy [8], Mitidieri [5] studied the strong convergence of solutions of (1.1).

The purpose of this paper is to continue the study initiated by Aizicovici, using the properties of almost nonexpansive curve, which was introduced by Djafari Rouhani [9]. In section 2, we describe the notations and contain some definitions and known results. Section 3 contains the several results [Theorem 1, 2, Corollary 1] concerning the asymptotic behavior of almost nonexpansive curve. Main results are given in Section 4. First we establish criterions for the weak convergence in $H$, as $t \rightarrow \infty$ of generalized solutions of (1.1) [Theorem 3, Corollary 3]. Next, we study the weak convergence of the Cesàro mean of the generalized solutions [Theorem 4].

\section{PRELIMINARIES.}

Let $H$ be a real Hilbert space with inner product $($,$) and norm \|\|$. Let $A$ be a maximal monotone (possibly multivalued) operator defined on subset $D(A) \subset H$.

As usual, we will put $[x, y] \in A \Leftrightarrow y \in A x$. We denote by $F$ the (possibly empty) set

$$
F=A^{-1} 0=\left\{x: x \in D(A), A^{0} x=0\right\}
$$

where $A^{0} y$ denotes the element of minimum norm in the closed convex set $A y$. Clearly, $F$ is a closed convex subset of $H$. For background material concerning maximal monotone operators, see [10], [11].

We will use " $w$-lim" or " - " to indicate weak convergence in $H$. The symbol $\bar{D}$ denotes the closure of the set $D$. For a function $u:[0, \infty) \rightarrow H$, we denote by $\omega_{w}(u(t))$ the weak $\omega$-limit set of $u$, i.e.,

$$
\omega_{w}(u(t))=\left\{y \in H: y=w-\lim u\left(t_{n}\right) \text { for some sequence } t_{n} \rightarrow \infty\right\}
$$

and by $\overline{c o} \omega_{w}(u(t))$ the closed convex hull of $\omega_{w}(u(t))$, respectively. Let $u:[0, \infty) \rightarrow H$ be a bounded function. With the function $u(t)$, we associate the functional

$$
\phi(y)=\limsup _{t \rightarrow \infty}\|u(t)-y\|^{2} .
$$

Then $\phi$ is a continuous, strictly convex function on $H$, satisfying $\phi(y) \rightarrow \infty$ as $\|y\| \rightarrow \infty$, and therefore $\phi$ has a unique minimum in $H$. The unique point $c \in H$ satisfying

$$
\phi(c)=\min _{\mathbf{y} \in \boldsymbol{H}} \phi(y)
$$

is called the asymptotic center of $u(t)$ and it is denoted by $c=A C(u(t))$.

Consider now the initial value problem (1.1), where $G$ satisfies (1.2), $x \in \overline{D(A)}$ and $f \in L_{l o c}^{1}([0, \infty): H)$. We recall the following definitions $([1],[4])$.

DEFINITION 1. A strong solution of $(1.1)$ on $[0, \infty)$ is a function $u \in W_{\text {loc }}^{\mathbf{1}, 1}([0, \infty): H)$ $\cap C([0, \infty): \overline{D(A)})$, satisfying $u(0)=x$ and $d u(t) / d t+A u(t)+G(u)(t) \ni f(t)$, a.e. on $[0, \infty)$.

DEFINITION 2. A function $u \in C([0, \infty): \overline{D(A)})$ is said to be a generalized solution to equation (1.1) if there are sequences $x_{n} \in \overline{D(A)}, f_{n} \in L_{l o c}^{1}([0, \infty): H)$ and $u_{n} \in C([0, \infty): H)$ such that $u_{n}$ is a strong solution of

$$
\begin{gathered}
\frac{d u_{n}}{d t}+A u_{n}+G\left(u_{n}\right) \ni f_{n} \\
u_{n}(0)=x_{n},
\end{gathered}
$$

$x_{n} \rightarrow x, f_{n} \rightarrow f$ in $L^{1}(0, T: H)$ and $u_{n} \rightarrow u$ in $C([0, T]: H)$, for each $0<T<\infty$. 
The following existence result is well-known ([1], [4]).

PROPOSITION 1. Let $G$ satisfy (1.2) and assume that:

(i) There exists $\gamma \in L_{l o c}^{1}([0, \infty): R)$ such that for every $u, v \in C([0, \infty): \overline{D(A)})$,

$$
\|G(u)-G(v)\|_{L^{1}(0, t: H)} \leq \int_{0}^{t} \gamma(s)\|u-v\|_{L^{\infty}(0, s: H)} d s, \quad 0 \leq s \leq t<\infty .
$$

(ii) For each $T \in(0, \infty)$, there is $\alpha_{T}:[0, \infty) \rightarrow[0, \infty)$ such that if $u \in C([0, T]: \overline{D(A)})$ is of bounded variation and $\|u\|_{L^{\infty}(0, T: H)} \leq R$, then

$$
\operatorname{var}(G(u):[0, t]) \leq \alpha_{T}(R)(1+\operatorname{var}(u:[0, t])), \quad 0 \leq t \leq T
$$

and

$$
\left\|G(u)\left(0^{+}\right)\right\| \leq \alpha_{T}(R) .
$$

Then,

(a) For each $x \in D(A)$ and $f \in B V_{\text {loc }}([0, \infty): H)$, problem (1.1) has a unique strong solution defined on $[0, \infty)$.

(b) For each $x \in \overline{D(A)}$ and $f \in L_{l o c}^{1}([0, \infty): H)$, problem (1.1) has a unique generalized solution defined on $[0, \infty)$.

\section{ASYMPTOTIC BEHAVIOR OF CURVES IN $\boldsymbol{H}$.}

In this section, we study asymptotic behavior of almost nonexpansive curve, which was introduced by Djafari Rouhani [9]. The following results are essentially in spirit of Djafari Rouhani. For the study and completeness, we give several results similar to those in [9] with detail and slightly different proofs.

Let $u \in C([0, \infty): H)$; in the sequel we refer to such $u$ as a curve in $H$. Let $\sigma(t)=(1 / t) \int_{0}^{t} u(\tau) d \tau$. We begin with the following:

DEFINITION 3 [9]. The curve $u(t)$ is almost nonexpansive (abbreviated ANEC) if for any $r, s, h \geq 0$,

where

$$
\|u(r+h)-u(s+h)\|^{2} \leq\|u(r)-u(s)\|^{2}+\epsilon(r, s),
$$

$$
\lim _{r, s \rightarrow \infty} \epsilon(r, s)=0 \text {. }
$$

REMARK 1. (a) A nonexpansive curve $\{u(t)\}$ (i.e., for any $r, s, h \geq 0, \| u(r+h)$ $-u(s+h)\|\leq\| u(r)-u(s) \|)$ is an ANEC.

(b) A bounded curve $\{u(t)\}$ that satisfies

$$
\|u(r+h)-u(s+h)\| \leq\|u(r)-u(s)\|+\epsilon_{1}(r, s)
$$

for any $r, s, h \geq 0$, where $\lim _{r, a \rightarrow \infty} \epsilon_{1}(r, s)=0$, is an ANEC.

In our next results, we will use the following notation:

$$
E(u(t))=\left\{q \in H: \lim _{t \rightarrow \infty}\|u(t)-q\| \text { exists }\right\} .
$$

Note that if $E(u(t)) \neq \emptyset$, then curve $\{u(t)\}$ is bounded.

LEMMA 1. Let $\{u(t)\}$ be an ANEC in $H$ and $p \in H$. Then for any $l, r \geq 0$,

$$
\begin{gathered}
2\left(u(l)-u(l+r), \frac{1}{t} \int_{0}^{t} u(\tau) d \tau-p\right) \\
\leq\|u(l)-p\|^{2}-\|u(l+r)-p\|^{2}+\frac{1}{t} \int_{0}^{r}\|u(l+r)-u(\tau)\|^{2} d \tau \\
\quad+\frac{1}{t} \int_{r}^{t} \epsilon(l, \tau-r) d \tau
\end{gathered}
$$


PROOF. Let $\sigma(t)=(1 / t) \int_{0}^{t} u(\tau) d \tau$. Note that for any $l, r \geq 0$,

$$
\begin{aligned}
& 2(u(l)-u(l+r), \sigma(t)-p) \\
= & \|u(l)-p\|^{2}-\|u(l+r)-p\|^{2}+\|u(l+r)-\sigma(t)\|^{2}-\|u(l)-\sigma(t)\|^{2} .
\end{aligned}
$$

Since

and

$$
\|u(l+r)-\sigma(t)\|^{2}=\frac{1}{t} \int_{0}^{t}\|u(l+r)-u(\tau)\|^{2} d \tau
$$

we obtain for any $l, r \geq 0$,

$$
\|u(l)-\sigma(t)\|^{2}=\frac{1}{t} \int_{0}^{t}\|u(l)-u(\tau)\|^{2} d \tau
$$

$$
\begin{aligned}
& \|u(l+r)-\sigma(t)\|^{2}-\|u(l)-\sigma(t)\|^{2} \\
& \leq \frac{1}{t} \int_{0}^{r}\|u(l+r)-u(\tau)\|^{2} d \tau+\frac{1}{t} \int_{r}^{t}\|u(l+r)-u(\tau)\|^{2} d \tau \\
& -\frac{1}{t} \int_{0}^{t-r}\|u(l)-u(\tau)\|^{2} d \tau \\
& =\frac{1}{t} \int_{0}^{r}\|u(l+r)-u(\tau)\|^{2} d \tau+\frac{1}{t} \int_{r}^{t}\left(\|u(l+r)-u(\tau)\|^{2}-\|u(l)-u(\tau-r)\|^{2}\right) d \tau \\
& \leq \frac{1}{t} \int_{0}^{r}\|u(l+r)-u(\tau)\|^{2} d \tau+\frac{1}{t} \int_{r}^{t} \epsilon(l, \tau-r) d \tau,
\end{aligned}
$$

and the proof is complete.

LEMMA 2. Let $\{u(t)\}$ be a bounded curve in $H$ and let $A C(u(t))$ be the asymptotic center of $\{u(t)\}$.

(a) Let $\left\{u\left(t_{n}\right)\right\}$ be a subnet of $\{u(t)\}$ and let $w-\lim _{n \rightarrow \infty} u\left(t_{n}\right)=p$. If $\lim _{t \rightarrow \infty}\|u(t)-p\|$ exists, then $p=A C(u(t))$.

(b) If $w-\lim _{t \rightarrow \infty} u(t)$ exists, then $w-\lim _{t \rightarrow \infty} u(t)=A C(u(t))$.

PROOF. Let $A C(u(t))=c$. Note that for $z \in H$,

$$
\begin{aligned}
\|u(t)-c\|^{2}=\|u(t)-z\|^{2} & +\|z-c\|^{2}+2(u(t)-z, z-c) \\
& \geq\|u(t)-z\|^{2}+2(u(t)-z, z-c) .
\end{aligned}
$$

To prove (a), let $w-\lim _{n \rightarrow \infty} u\left(t_{n}\right)=p$ and $\{\|u(t)-p\|\}$ be convergent. Letting $t \rightarrow \infty$ in (3.1) with $z$ replaced by $p$, we have

$$
\phi(c) \geq \lim _{t \rightarrow \infty}\|u(t)-p\|^{2}+2 \limsup _{t \rightarrow \infty}(u(t)-p, p-c) .
$$

Since $\lim \sup _{t \rightarrow \infty}(u(t)-p, p-c) \geq \lim _{n \rightarrow \infty}\left(u\left(t_{n}\right)-p, p-c\right)=0$, we have

$$
\phi(c) \geq \lim _{t \rightarrow \infty}\|u(t)-p\|^{2}
$$

i.e., $\phi(c) \geq \phi(p)$. Thus it follows from definition of $c$ that $c=p$.

To prove (b), let $w-\lim _{t \rightarrow \infty} u(t)=q$. Letting $t \rightarrow \infty$ in (3.1) with $z$ replaced by $q$, we have

$$
\phi(c) \geq \limsup _{t \rightarrow \infty}\|u(t)-q\|^{2}+2 \lim _{t \rightarrow \infty}(u(t)-q, q-c)=\phi(q) .
$$

This implies that $c=q$.

LEMMA 3 [9]. Let $\{u(t)\}$ be an $A N E C$ in $H$. If 


$$
w-\lim _{t \rightarrow \infty}(u(t+h)-u(t))=0
$$

for any $h \geq 0$, then $\omega_{w}(u(t)) \subset E(u(t))$. In particular, if $\omega_{w}(u(t)) \neq \emptyset$, then $\{u(t)\}$ is bounded.

PROOF. Let $p \in \omega_{w}(u(t))$. Then there exists a sequence $t_{n} \rightarrow \infty$ such that $w-\lim _{n \rightarrow \infty} u\left(t_{n}\right)=p$. On the other hand, by Lemma 1 , we have the inequality

$$
\begin{aligned}
& 2\left(u(r)-u(r+s), \frac{1}{t} \int_{0}^{t} u\left(t_{n}+\tau\right) d \tau-p\right) \\
& \leq\|u(r)-p\|^{2}-\|u(r+s)-p\|^{2}+\frac{1}{t} \int_{0}^{s}\left\|u(r+s)-u\left(t_{n}+\tau\right)\right\|^{2} d \tau \\
& \quad+\frac{1}{t} \int_{s}^{t} \epsilon\left(r, t_{n}+\tau-s\right) d \tau \\
& \leq\|u(r)-p\|^{2}-\|u(r+s)-p\|^{2}+\frac{M(s, r)}{t}+\frac{1}{t} \int_{s}^{t} \epsilon\left(r, t_{n}+\tau-s\right) d \tau
\end{aligned}
$$

for any $r, s \geq 0, t>0, n \geq 1$, where $M(s, r)$ is a constant depending only on $s$ and $r$. Let $\epsilon>0$ be given, and choose $l>0$ so that

$$
\epsilon(h, t) \leq \epsilon
$$

for all $h, t \geq l$. Then, taking $r \geq l, s \geq 0, t>0$ fixed, and letting $n \rightarrow \infty$, i.e., $t_{n} \rightarrow \infty$, we have

$$
0 \leq\|u(r)-p\|^{2}-\|u(r+s)-p\|^{2}+\frac{M(s, r)}{t}+\epsilon
$$

for any $r \geq l, s \geq 0, t>0$. Now letting $t \rightarrow \infty$, we obtain

$$
\|u(r+s)-p\|^{2} \leq\|u(r)-p\|^{2}+\epsilon
$$

for any $r \geq l$ and $s \geq 0$. Hence

$$
\limsup _{t \rightarrow \infty}\|u(t)-p\|^{2} \leq\|u(r)-p\|^{2}+\epsilon
$$

for any $r \geq l$. Thus

$$
\limsup _{t \rightarrow \infty}\|u(t)-p\|^{2} \leq \liminf _{t \rightarrow \infty}\|u(t)-p\|^{2}+\epsilon
$$

which implies that $\lim _{t \rightarrow \infty}\|u(t)-p\|^{2}$ exists and hence $p \in E(u(t))$.

THEOREM 1. Let $\{u(t)\}$ be an $A N E C$ in $H$. Then the following are equivalent:

(i) $w-\lim _{t \rightarrow \infty} u(t)$ exists.

(ii) $E(u(t)) \neq \emptyset$ and $\omega_{w}(u(t)) \subset E(u(t))$.

Moreover, if $w-\lim _{t \rightarrow \infty} u(t)$ exists, then it is the asymptotic center of $\{u(t)\}$.

PROOF. Suppose that $w-\lim _{t \rightarrow \infty} u(t)$ exists. Then $\omega_{w}(u(t)) \neq \emptyset$ and

$$
w-\lim _{t \rightarrow \infty}(u(t+h)-u(t))=0
$$

for any $h \geq 0$. It follows from Lemma 3 that $\omega_{w}(u(t)) \subset E(u(t))$, and hence (i) implies (ii). To show that (ii) implies (i), let $E(u(t)) \neq \emptyset$ and $\omega_{w}(u(t)) \subset E(u(t))$. By $E(u(t)) \neq \emptyset,\{u(t)\}$ is bounded. Then since a Hilbert space is reflexive, $\{u(t)\}$ has a subnet $\left\{u\left(t_{n}\right)\right\}$ which converges weakly to some $p \in H$. By $\omega_{w}(u(t)) \subset E(u(t)),\{\|u(t)-p\|\}$ is convergent. It follows from (a) of Lemma 2 that $p=A C(u(t))$. This means that $\omega_{w}(u(t))=\{A C(u(t))\}$. Thus every weakly convergent subnet of $\{u(t)\}$ converges weakly to $A C(u(t))$ and hence $\omega-\lim _{t \rightarrow \infty} u(t)=A C(u(t))$.

As a direct consequence of Lemma 3 and Theorem 1, we have the following:

COROLLARY 1. Let $\{u(t)\}$ be an $A N E C$. Then the following condition 
(iii) $E(u(t)) \neq \emptyset$ and $w-\lim _{t \rightarrow \infty}(u(t+h)-u(t))=0$ for any $h \geq 0$ is equivalent to each one of conditions (i) and (ii) in Theorem 1.

We turn now to the weak convergence of Cesàro mean $\sigma(t)$ of curve $\{u(t)\}$. The following lemma is the principal ingredient.

LEMMA 4. Let $\{u(t)\}$ be an $A N E C$. Then

$$
\omega_{w}(\sigma(t)) \subset E(u(t))
$$

PROOF. Let $p \in \omega_{w}(\sigma(t))$. Then there exists a sequence $t_{n} \rightarrow \infty$ such that $w-\lim _{n \rightarrow \infty} \sigma\left(t_{n}\right)=p$. By Lemma 1 , we have the inequality

$$
\begin{gathered}
2\left(u(r)-u(r+s), \sigma\left(t_{n}\right)-p\right) \\
\leq\|u(r)-p\|^{2}-\|u(r+s)-p\|^{2}+\frac{1}{t_{n}} \int_{0}^{s}\|u(r+s)-u(\tau)\|^{2} d \tau \\
+\frac{1}{t_{n}} \int_{s}^{t_{n}} \epsilon(r, \tau-s) d \tau
\end{gathered}
$$

for any $r, s \geq 0$. Let $\epsilon>0$ be given and choose $l>0$ so that

$$
\epsilon(h, t) \leq \epsilon
$$

for all $h, t \geq l$. Then taking $r \geq l, s \geq 0$ fixed and letting $t_{n} \rightarrow \infty$, we obtain

$$
\|u(r+s)-p\|^{2} \leq\|u(r)-p\|^{2}+\epsilon
$$

Therefore by the same argument as in Lemma 3, we conclude that $\lim _{t \rightarrow \infty}\|u(t)-p\|^{2}$ exists and hence $p \in E(u(t))$.

We also prepare a lemma; see also [12].

LEMMA 5. $E(u(t)) \cap \cap_{s} \geq 0 \overline{c o}\{u(t): t \geq s\}$ contains at most a singleton. If $E(u(t)) \cap \cap_{s} \geq 0 \overline{c o}$ $\{u(t): t \geq s\} \neq \emptyset$, then

$$
\{A C(u(t))\}=E(u(t)) \cap \bigcap_{s \geq 0} \overline{c o}\{u(t): t \geq s\} .
$$

PROOF. Note that $\bigcap_{s \geq 0} \overline{c o}\{u(t): t \geq s\}=\overline{c o} \omega_{w}(u(t))$; see [13]. Let $q_{1}, q_{2} \in \omega_{w}(u(t))$ and $p_{1}, p_{2} \in E(u(t))$. Then we have

$$
\lim _{t \rightarrow \infty}\left\|u(t)-p_{\imath}\right\|^{2}=\phi\left(p_{\imath}\right)
$$

for $i=1,2$ and

$$
\left\|u(t)-p_{2}\right\|^{2}=\left\|u(t)-p_{1}\right\|^{2}+\left\|p_{1}-p_{2}\right\|^{2}+2\left(u(t)-p_{1}, p_{1}-p_{2}\right) .
$$

Thus

$$
\begin{aligned}
& \phi\left(p_{2}\right)=\phi\left(p_{1}\right)+\left\|p_{1}-p_{2}\right\|^{2}+2\left(q_{1}-p_{1}, p_{1}-p_{2}\right) \\
& \phi\left(p_{2}\right)=\phi\left(p_{1}\right)+\left\|p_{1}-p_{2}\right\|^{2}+2\left(q_{2}-p_{1}, p_{1}-p_{2}\right) .
\end{aligned}
$$

Hence by subtraction, we have $\left(q_{1}-q_{2}, p_{1}-p_{2}\right)=0$. This result extends obviously to every $q_{1}, q_{2} \in \overline{c o} \omega_{w}(u(t))$. Therefore it follows that if $p_{1}, p_{2} \in E(u(t)) \cap \overline{c o} \omega_{w}(u(t))$, then $p_{1}=p_{2}$, and hence

$$
E(u(t)) \cap \bigcap_{s \geq 0} \overline{c o}\{u(t): t \geq s\}=E(u(t)) \cap \overline{c o} \omega_{w}(u(t))
$$

is at most a singleton. Let $\{p\}=E(u(t)) \cap \bigcap_{s \geq 0} \overline{c o}\{u(t): t \geq s\}$. Since $p \in E(u(t))$, we have $\lim _{t \rightarrow \infty}\|u(t)-p\|^{2}=\phi(p)$. 
Let $q \in \omega_{w}(u(t)), q=w-\lim _{t_{k} \rightarrow \infty} u\left(t_{k}\right)$ and let $u \in H$. Passing to the limit as $t_{k} \rightarrow \infty$ in

$$
\left\|u\left(t_{k}\right)-u\right\|^{2}=\left\|u\left(t_{k}\right)-p\right\|^{2}+\|p-u\|^{2}+2\left(u\left(t_{k}\right)-p, p-u\right),
$$

we find

$$
\phi(u) \geq \phi(p)+2(q-p, p-u) .
$$

This inequality holds for all $q \in \omega_{w}(u(t))$, and hence, also for all $q \in \overline{c o} \omega_{w}(u(t))$. Since $p \in \overline{c o} \omega_{w}(u(t))$, we obtain

$$
\phi(u) \geq \phi(p)
$$

for all $u \in H$. This implies that $p=A C(u(t))$.

THEOREM 2. Let $\{u(t)\}$ be an $A N E C$ in $H$. Then the following are equivalent:

(i) $w-\lim _{t \rightarrow \infty} \sigma(t)$ exists.

(ii) $E(u(t)) \neq \emptyset$.

Moreover, if $w-\lim _{t \rightarrow \infty} \sigma(t)$ exists, then it is the asymptotic center of $\{u(t)\}$.

PROOF. Suppose that $w-\lim _{t \rightarrow \infty} \sigma(t)$ exists. Then $\omega_{w}(\sigma(t)) \neq \emptyset$. It follows from Lemma 4 that $\omega_{w}(\sigma(t)) \subset E(u(t))$ and hence (i) implies (ii). To show that (ii) implies (i), let $E(u(t)) \neq \emptyset$. Then $\{u(t)\}$ is bounded, and hence $\{\sigma(t)\}$ is also bounded. Then since a Hilbert space is reflexive, $\{\sigma(t)\}$ has a subnet $\left\{\sigma\left(t_{n}\right)\right\}$ which converges weakly to some $p \in H$. By Lemma 4, $\omega_{w}(\sigma(t)) \subset E(u(t))$, and so $p \in E(u(t))$. If there was another subnet $\left\{\sigma\left(t_{l}\right)\right\}$ which converges weakly to some $q \in H$, then we also have $q \in E(u(t))$. Hence the net

$$
2(u(t), q-p)+\|p\|^{2}-\|q\|^{2}=\|u(t)-p\|^{2}-\|u(t)-q\|^{2}
$$

has a limit as $t \rightarrow \infty$, i.e., $\lim _{t \rightarrow \infty}(u(t), q-p)$ exists. Therefore $(p, q-p)=(q, q-p)$, which implies $\|p-q\|^{2}=0$, and hence $p=q$. Hence every weakly convergent subnet of $\sigma(t)$ converges weakly to $p$, and hence $w-\lim _{t \rightarrow \infty} \sigma(t)=p$. We obviously have $p \in E(u(t)) \cap \cap_{s} \geq 0^{\overline{c o}}$ $\{u(t): t \geq s\}=E(u(t)) \cap \overline{c o} \omega_{w}(u(t))$. Therefore it follows from Lemma 5 that $p$ is the asymptotic center of $\{u(t)\}$.

\section{ASYMPTOTIC BEHAVIOR OF SOLUTIONS IN $\boldsymbol{H}$.}

In this section, we give the main results concerning the asymptotic behavior, as $t \rightarrow \infty$ of generalized solutions of (1.1). Following Aizicovici [1], we assume the following conditions:

(C1) $G$ satisfies (1.2), (2.1) and (2.2).

(C2) For every $u, v \in C([0, \infty): \overline{D(A)})$,

$$
\int_{s}^{t}(G(u)(\tau)-G(v)(\tau), u(\tau)-v(\tau)) d \tau \geq 0, \quad 0 \leq s \leq t<\infty .
$$

(C3) $G(v) \in L^{1}(0, \infty: H)$ for each constant function $v(t) \equiv v \in D(A)$.

(C4) $f \in L^{1}(0, \infty: H)$.

(C5) $x \in \overline{D(A)}$.

We begin with a simple lemma which will play a crucial role in our results.

LEMMA 6. Let $A$ be a maximal monotone operator on $H$. Assume that (C1) holds. Let $f$, $\widehat{f} \in L_{\text {loc }}^{1}([0, \infty): H)$ and $x, \widehat{x} \in \overline{D(A)}$. Let $u, \widehat{u}$ be the corresponding generalized solutions of $(1.1)$. If $(\mathrm{C} 2)$ is satisfied, then

$$
\|u(t)-\widehat{u}(t)\| \leq\|u(s)-\widehat{u}(s)\|+\int_{s}^{t}\|f(\tau)-\widehat{f}(\tau)\| d \tau
$$

for $0 \leq s \leq t<\infty$.

PROOF. Taking into account definitions 1 and 2 , as well as Proposition 1 , it clearly suffices to prove (4.1) in the case that $u$ and $\widehat{u}$ are strong solutions of (1.1). By the monotonicity of $A$, 
we have

$$
\begin{gathered}
0 \leq\left(f(t)-u^{\prime}(t)-G(u)(t)-\widehat{f}(t)+\widehat{u}^{\prime}(t)+G(\widehat{u})(t), u(t)-\widehat{u}(t)\right) \\
=(f(t)-\widehat{f}(t), u(t)-\widehat{u}(t))-\left(u^{\prime}(t)-\widehat{u}^{\prime}(t), u(t)-\widehat{u}(t)\right) \\
-(G(u)(t)-G(\widehat{u})(t), u(t)-\widehat{u}(t)) \\
\leq\|f(t)-\widehat{f}(t)\|\|u(t)-\widehat{u}(t)\|-\frac{1}{2} \frac{d}{d t}\|u(t)-\widehat{u}(t)\|^{2} \\
-(G(u)(t)-G(\widehat{u})(t), u(t)-\widehat{u}(t))
\end{gathered}
$$

for $t>0$. Integrating on $[s, t]$, it follows from (C2) that

$$
\frac{1}{2}\|u(t)-\widehat{u}(t)\|^{2} \leq \frac{1}{2}\|u(s)-\widehat{u}(s)\|^{2}+\int_{s}^{t}\|f(\tau)-\widehat{f}(\tau)\|\|u(\tau)-\widehat{u}(\tau)\| d \tau .
$$

The inequality (4.1) follows from (4.2) by Gronwall's lemma (see [4, Lemma A.5]).

COROLLARY 2. Let $A$ be a maximal monotone operator on $H$, and $f \in L_{l o c}^{1}([0, \infty): H)$. Assume that (C1), (C2), (C3) and (C5) hold. If $u$ is a generalized solution of (1.1), then

$$
\|u(r+h)-u(s+h)\| \leq\|u(r)-u(s)\|+\int_{s}^{s+h}\|f(\tau+(r-s))-f(\tau)\| d \tau .
$$

PROOF. It is enough to apply Lemma 6 with $\widehat{f}(t)=f(t+(r-s))$ and $\widehat{u}(t)=u(t+(r-s))$.

PROPOSITION 2. Let $A$ be a maximal monotone operator on $H$. Assume that (C1), (C2), (C3), (C4) and (C5) hold. If $u$ is a generalized solution of $(1.1)$ and if $\{u(t)\}$ is bounded on $[0, \infty)$, then the curve $\{u(t)\}$ is an $A N E C$ in $H$.

PROOF. By (C4), we have

$$
\lim _{\substack{s, r \rightarrow \infty \\ r>s}} \int_{s}^{\infty}\|f(\tau+(r-s))-f(\tau)\| d \tau=0 .
$$

Thus the result follows from Corollary 2 and Remark 1 (b) by taking

$$
\epsilon_{1}(r, s)= \begin{cases}\int_{r}^{\infty}\|f(\tau+(r-s))-f(\tau)\| d \tau & \text { if } r \geq s \\ \int_{r}^{\infty}\|f(\tau+(r-s))-f(\tau)\| d \tau & \text { if } s \geq r .\end{cases}
$$

Now, we apply Theorem 1 and 2 to study the asymptotic behavior of the generalized solution $u$ of (1.1). We need the next known result.

LEMMA 7 [1]. Let $A$ be a maximal monotone operator on $H$. Assume that (C1), (C2), (C3) and (C5) hold. Let $u$ be the generalized solution of (1.1) corresponding to $f \in L_{l o c}^{1}([0, \infty): H)$. Then

$$
\|u(t)-y\| \leq\|u(s)-y\|+\int_{s}^{t}\|f(\tau)-G(y)(\tau)-z\| d \tau
$$

whenever $0 \leq s \leq t<\infty$ and $z \in A y$.

The following is also necessary. We contain the proof for the completeness (see also [1]).

LEMMA 8. Let $A$ be a maximal monotone operator on $H$. Assume that (C1), (C2), (C3), (C4) and (C5) hold. Let $u$ be a generalized solution of (1.1). Then $F \subset E(u(t))$.

PROOF. Let $y \in F$. By (4.3) in Lemma 7,

$$
\|u(t)-y\| \leq\|x-y\|+\int_{0}^{t}\|f(\tau)-G(y)(\tau)\| d \tau
$$

for $t \geq 0$. Thus, using (C3) and (C4), we deduce that $\{u(t)\}$ is bounded on $[0, \infty)$. Also it follows from (4.3) in Lemma 7 that

$$
\begin{aligned}
\|u(t)-y\|- & \int_{0}^{t}\|f(\tau)-G(y)(\tau)\| d \tau \\
& \leq\|u(s)-y\|-\int_{0}^{s}\|f(\tau)-G(y)(\tau)\| d \tau, \quad 0 \leq s \leq t<\infty
\end{aligned}
$$


This (4.4), (C3), (C4) and the boundedness of $u$ imply that the function $\psi(t)=\|u(t)-y\|-\int_{0}^{t}\|f(\tau)-G(y)(\tau)\| d \tau$ is bounded on $[0, \infty)$. By (4.4), it is also nonincreasing. Therefore, again, taking into account (C3) and (C4), we have that $\lim _{t \rightarrow \infty}\|u(t)-y\|$ exists and hence $y \in E(u(t))$.

THEOREM 3. Let $A$ be a maximal monotone operator on $H$. Assume that (C1), (C2), (C3), (C4) and (C5) hold. Let $u$ be a generalized solution of (1.1). Then the following are equivalent:

(i) $w-\lim u(t)$ exists.

(ii) $E(u(t)) \neq \emptyset$ and $\omega_{w}(u(t)) \subset E(u(t))$.

PROOF. Since $\{u(t)\}$ is bounded under each condition of (i) and (ii), $\{u(t)\}$ is an $A N E C$ by Proposition 2. Thus the result follows from Theorem 1.

COROLLARY 3. Let $A$ be a maximal monotone operator on $H$. Assume that (C1), (C2), (C3), (C4) and (C5) hold. Let $u$ be a generalized solution of (1.1). Then the following condition

(iii) $E(u(t)) \neq \emptyset$ and $w-\lim _{t \rightarrow \infty}(u(t+h)-u(t))=0$ for any $h \geq 0$ is equivalent to each one of conditions (i) and (ii) in Theorem 3.

PROOF. The result follows from Corollary 1 and Theorem 3.

As a direct consequence, we have the following:

COROLLARY 4. Let $A$ be a maximal monotone operator on $H$. Assume that (C1), (C2), (C3), (C4) and (C5) hold. Let $u$ be a generalized solution of (1.1). If $F \neq \emptyset$ and $w-\lim _{t \rightarrow \infty}(u(t+h)-u(t))=0$ for any $h>0$, then $u(t)$ converges weakly as $t \rightarrow \infty$ to the asymptotic center of the curve $\{u(t)\}$.

PROOF. By Lemma 8, we have $F \subset E(u(t))$. Thus the result follows from Theorem 1 and Corollary 3.

THEOREM 4. Let $A$ be a maximal monotone operator on $H$. Assume that (C1), (C2), (C3), (C4) and (C5) hold. Let $u$ be a generalized solution of (1.1), and $\sigma(t)=\frac{1}{t} \int_{0}^{t} u(\tau) d \tau$. If $E(u(t)) \neq \emptyset$, then $\sigma(t)$ converges weakly as $t \rightarrow \infty$ to the asymptotic center of the curve $\{u(t)\}$.

PROOF. Since $\{u(t)\}$ is bounded by $E(u(t)) \neq \emptyset,\{u(t)\}$ is an $A N E C$ by Proposition 2. Thus the result follows from Theorem 2 .

As a consequence, we also have the following:

COROLLARY 5. Let $A$ be a maximal monotone operator on $H$. Assume that (C1), (C2), (C3), (C4) and (C5) hold. Let $u$ be a generalized solution of $(1.1)$ and $\sigma(t)=\frac{1}{t} \int_{0}^{t} u(\tau) d \tau$. If

$F \neq \emptyset$, then $\sigma(t)$ converges weakly as $t \rightarrow \infty$ to the asymptotic center of the curve $\{u(t)\}$.

PROOF. By Lemma 8, we have $F \subset E(u(t))$. Thus the result follows from Theorem 4.

REMARK 2. (a) Under the hypotheses of Theorem 3, the fact that the following condition

(iv) $F \neq \emptyset$ and $\omega_{w}(u(t)) \subset F$ is

is equivalent to (i) in Theorem 3 was proved by Aizicovici [1]. Consequently, all the conditions (i) and (ii) in Theorem 3, (iii) in Corollary 3 and this (iv) are equivalent.

(b) The case in which $G \equiv 0$ was previously considered by Moroşanu [14] and Djafari Rouhani [9]. Theorem 3 is a new result even in the case in which $G \equiv 0$.

(c) Properties of the metric projection were not used in Theorem 4 in contrast to ([1, Theorem 2.3]).

(d) As in [1], [5], [6], [15], [16], our results can be used to study the asymptotic behavior of solutions of the related nonlinear Volterra equation: 


$$
\begin{aligned}
& \text { J.S. JUNG, J.Y. PARK AND H.J. KANG } \\
& u(t)+\int_{0}^{t} b(t-s) A u(s) d s \ni g(t), \quad t>0 \\
& u(0)=x=g(0) .
\end{aligned}
$$

ACKNOWLEDGEMENT. The present study was supported by the Basic Science Research Institute Program, Ministry of Education, 1992, Project No. BSRI-92-103.

\section{REFERENCES}

1. AIZICOVICI, S., On the asymptotic behavior of the solutions of Volterra equations in Hilbert space, Nonlinear Anal. 3 (1983), 271-278.

2. CLEMENT, Ph., MacCAMY, R.C. and NOHEL, J.A., Asymptotic properties of solutions of nonlinear abstract Volterra equations, J. Integral Equations 3 (1981), 185-216.

3. CLEMANT, $\mathrm{Ph}$. and NOHEL, J.A., Abstract linear and nonlinear Volterra equations in preserving positivity, SIAM J. Math. Anal. 10 (1979), 336-338.

4. CRANDALL, M.G. and NOHEL, J.A., An abstract functional differential equation and a related nonlinear Volterra equation, Israel J. Math. 29 (1978), 313-328.

5. MITIDIERI, E., Asymptotic behavior of the solutions of a class of functional differential equation: Remark on a related Volterra equation, J. Math. Anal. Appl. 107 (1985), 211-221.

6. NOHEL, J.A., Nonlinear Volterra equations for heat flow in materials with memory, in Integral and Functional Differential Equations, Lecture Notes in Pure Appl. Math. 67, Dekkar, New York, 1981.

7. RENNOLET, C., Existence and boundedness results for abstract nonlinear Volterra equations of nonconvolution type, J. Integral Equations 3 (1981), 137-151.

8. PAZY, A., Strong convergence of semigroups of nonlinear contraction in Hilbert spaces, $J$. Anal. Math. 36 (1978), 1-35.

9. DJAFARI ROUHANI, B., Asymptotic behavior of quasi-autonomous dissipative systems in Hilbert spaces, J. Math. Anal. Appl. 147 (1990), 465-476.

10. BARBU, V., Nonlinear Semigroups and Differential Equations in Banach spaces, Editura Academiei R.S.R., București, 1976.

11. BRÉZIS, H., Opérateurs Maximaux Monotones et Semigroupes de Contractions dans les Espaces de Hilbert, North Holland, 1973.

12. PAZY, A., Remarks on nonlinear ergodic theory in Hilbert space, Nonlinear Anal. 3 (1979), 863-871.

13. BRUCK, R., On the almost convergence of iterates of a nonexpansive mapping in a Hilbert space and the structure of the weak $\omega$-limit set, Israel J. Math. 29 (1987), 1-17.

14. MOROSANU, G., Asymptotic behavior of solutions of differential equations associated to monotone operators, Nonlinear Anal. 3 (1979), 873-883.

15. BAILLON, J.B. and CLEMENT, Ph., Ergodic theorems for nonlinear Volterra equations in Hilbert space, Nonlinear Anal. 5 (1981), 789-801.

16. HIRANO, N., Asymptotic behavior of solutions of nonlinear Volterra equations, J. Diff. Equations 47 (1983), 163-179. 


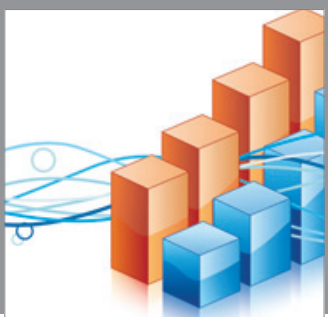

Advances in

Operations Research

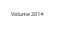

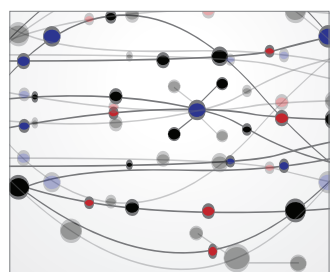

\section{The Scientific} World Journal
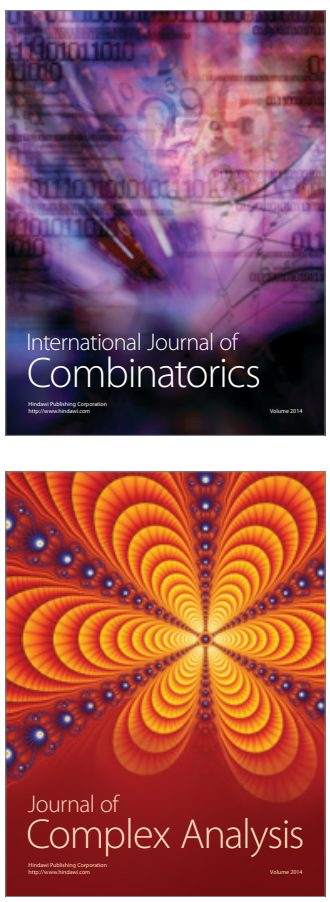

International Journal of

Mathematics and

Mathematical

Sciences
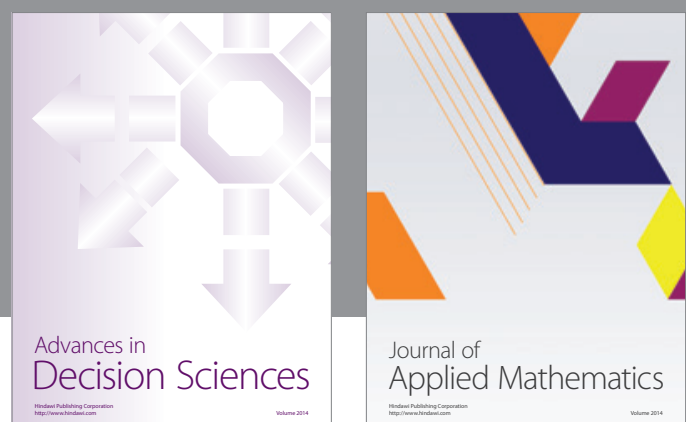

Journal of

Applied Mathematics
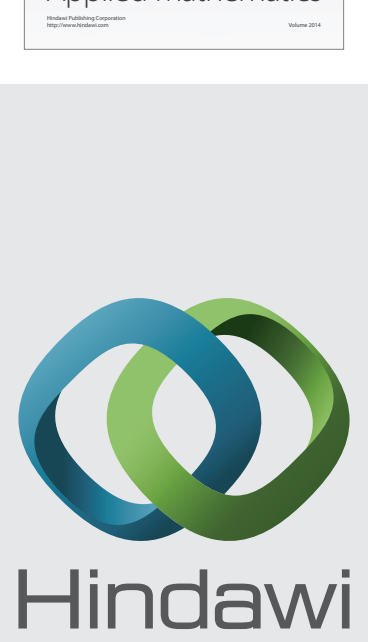

Submit your manuscripts at http://www.hindawi.com
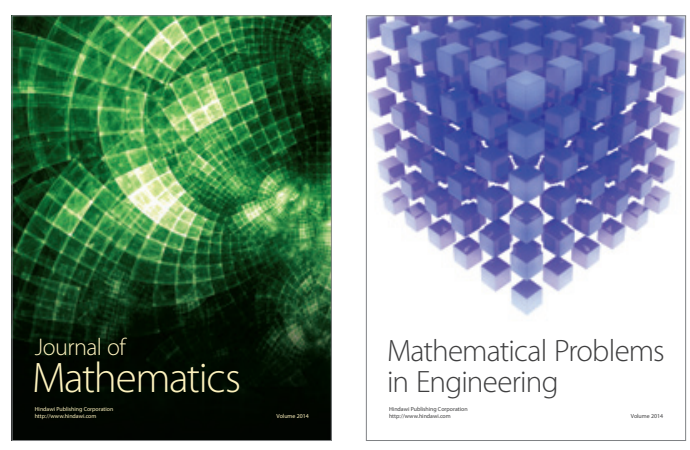

Mathematical Problems in Engineering
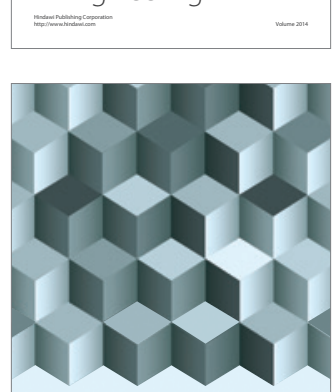

Journal of

Function Spaces
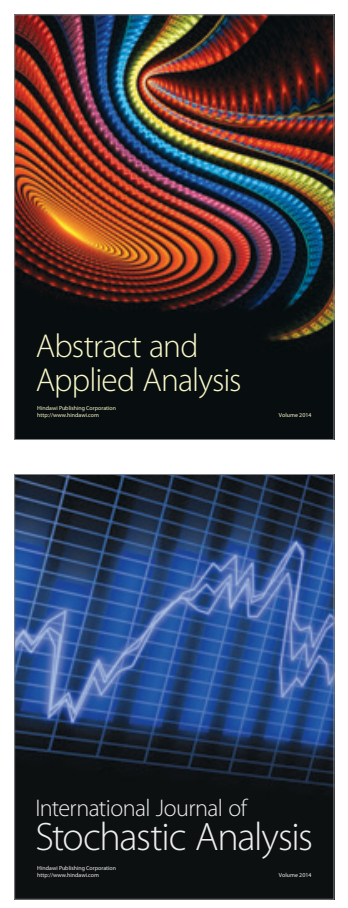

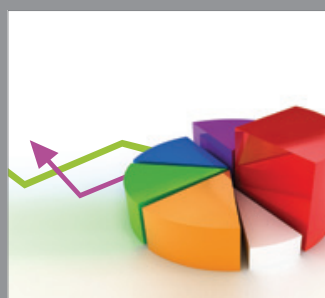

ournal of

Probability and Statistics

Promensencen
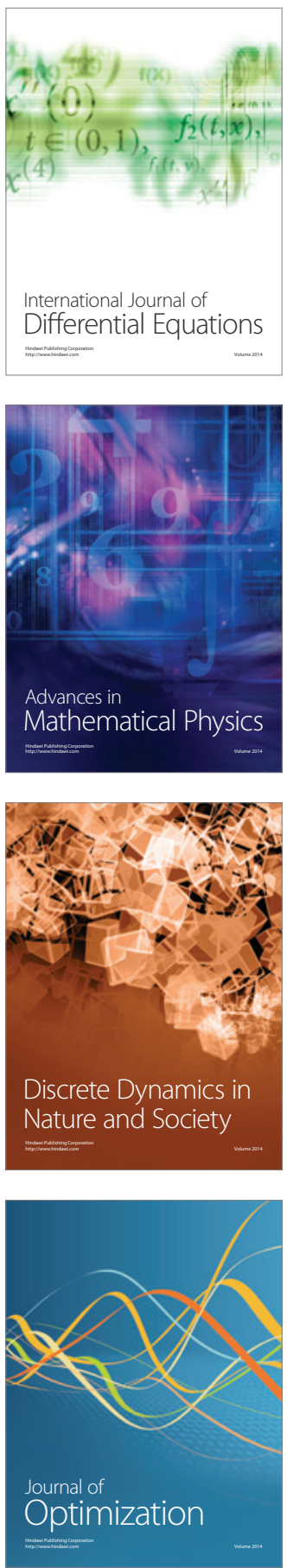